Pacific Journal of Mathematics

MULTIPLICITY TYPE AND SUBALGEBRA STRUCTURE IN 


\title{
MULTIPLICITY TYPE AND SUBALGEBRA STRUCTURE IN UNIVERSAL ALGEBRAS
}

\author{
MATTHEW GOULD
}

\begin{abstract}
By a universal algebra, or briefly, an algebra we shall here mean a pair $\langle A ; F\rangle$ consisting of a nonvoid set $A$ and a nonvoid set $F$ of finitary operations on $A$. The multiplicity type of $\langle A ; F\rangle$ is the sequence $\mu=\left\langle\mu_{0}, \mu_{1}, \cdots, \mu_{n}, \cdots\right\rangle$ where $\mu_{n}$ is the cardinality of $\{f \in F \mid f$ is $n$-ary $\}$. The class of all algebras of multiplicity type $\mu$ is denoted $K(\mu)$.

We shall study the relationship between the multiplicity type of an algebra and its family of subalgebras. To this end, we set $S(A ; F)=\{B \mid \phi \neq B \cong A$ and $\langle B ; F\rangle$ is a subalgebra of $\langle A ; F\rangle\}$ and, for every multiplicity type $\mu, T(\mu)=$ $\{S(A ; F) \mid\langle A ; F\rangle \in K(\mu)\}$. We define a quasi-ordering $\leqq$ and an equivalence $\equiv$ on the class of multiplicity types as follows. If $\mu$ and $\mu^{\prime}$ are multiplicity types, define $\mu \leqq \mu^{\prime}$ if $T(\mu) \cong T\left(\mu^{\prime}\right)$ and $\mu \equiv \mu^{\prime}$ if $T(\mu)=T\left(\mu^{\prime}\right)$. We shall give necessary and sufficient conditions for $\mu \leqq \mu^{\prime}$, in terms of properties of cardinal numbers, and we shall also find a "normal form" for multiplicity types, whereby every multiplicity type will have a unique representation in normal form and the ordering of multiplicity types in normal form will be characterized by relatively simple criteria.
\end{abstract}

Our major results, those which characterize the ordering and establish normal form, are Theorems 2.1, 2.2, 2.3, and 2.4.

A family $\mathfrak{U}$ of subsets of a set $A$ is called a restricted closure system if whenever $\mathfrak{B} \subseteq \mathfrak{U}$ and $\bigcap(X \mid X \in \mathfrak{B})$ is nonvoid, then $\bigcap(X \mid X \in \mathfrak{B}) \in \mathfrak{A}$. If $B \cong A$ then the closure of $B$, denoted [B], is defined to be $\cap(X \mid X \in \mathfrak{A}, X \supseteqq B)$, provided this intersection is not void. For any algebra $\langle A ; F\rangle$ it is easily seen that $S(A ; F)$ is a restricted closure system. Birkhoff and Frink [1] proved that for any family $\mathfrak{A}$ of subsets of a nonvoid set $A$, there is an algebra $\langle A ; F\rangle$ such that $\mathfrak{U}=S(A ; F)$, if and only if $\mathfrak{U}$ is an algebraic closure system, that is, a restricted closure system which is closed under directed union. We shall give a similar result (Theorem 1.1) with a restriction on the multiplicity type.

One minor result of particular interest is the fact that the subalgebra family of any algebra whose operations are finite in number can be realized as the subalgebra family of an algebra having precisely one operation. This is a consequence of Lemma 2.1.

A word on notation: $\mu$ and $\mu^{\prime}$ will always denote multiplicity types, and the cardinality of a set $A$ will be denoted $|A|$. 
1. Preliminary results. In this section we shall establish several results which will be helpful in proving the later characterization theorems for the ordering $\leqq$ among multiplicity types. For the most part, these are simply technical lemmas of no interest for their own sake. The main result is Theorem 1.2, which gives necessary conditions for $\mu \leqq \mu^{\prime}$. In $\S 2$, these conditions will be shown to be sufficient when all entries of $\mu$ are countable.

A. Definitions and simple lemmas. The following definitions are largely for the purpose of establishing convenient notation.

Definition. If $\mu$ and $\mu^{\prime}$ are multiplicity types we say that $\mu^{\prime}$ accepts $\mu$ provided $\mu_{0}=0$ implies $\mu_{0}^{\prime}=0$. If $\mu^{\prime}$ accepts $\mu$ and $\mu$ accepts $\mu^{\prime}$, then $\mu$ and $\mu^{\prime}$ are termed compatible.

DEFINITION. Let $n$ be a natural number and $\mathfrak{m}$ a nonzero cardinal. We shall denote by $\varepsilon_{n}(\mathfrak{m})$ the multiplicity type having $\mathfrak{m}$ as its $n^{\text {th }}$ entry and zeroes everywhere else. The multiplicity type $\bar{\varepsilon}_{n}(\mathfrak{m})$ is defined as follows. If $n=0$, then $\bar{\varepsilon}_{n}(\mathfrak{m})=\varepsilon_{n}(\mathfrak{m})$. If $n>0$, then $\left(\bar{\varepsilon}_{n}(\mathfrak{m})\right)_{0}=1,\left(\bar{\varepsilon}_{n}(\mathfrak{m})\right)_{n}=\mathfrak{m}$, and $\left(\bar{\varepsilon}_{n}(\mathfrak{m})\right)_{k}=0$ for $k \neq 0, n$. For simplicity, $\varepsilon_{n}(1)$ and $\bar{\varepsilon}_{n}(1)$ will be denoted simply by $\varepsilon_{n}$ and $\bar{\varepsilon}_{n}$, respectively.

Definition. If $\mu$ and $\mu^{\prime}$ are multiplicity types, we define $\mu+\mu^{\prime}$ to be their pointwise sum; that is, $\left(\mu+\mu^{\prime}\right)_{k}=\mu_{k}+\mu_{k}^{\prime}$ for every $k$. Similarly, we define the sum of any set of multiplicity types.

Definition. The length of $\mu$, denoted $l(\mu)$, is the greatest integer $n$ such that $\mu_{n} \neq 0$. If no such integer exists, we set $l(\mu)=\infty$. We denote by $s(\mu)$ the sum of the entries of $\mu$; that is, $s(\mu)=\sum\left(\mu_{k} \mid k \geqq 0\right)$.

The following lemma establishes simple properties of the ordering.

Lemma 1.1. ( i ) If $\mu \leqq \mu^{\prime}$, then $\mu^{\prime}$ accepts $\mu$.

(ii) If $\mu^{\prime}$ accepts $\mu$ and $\mu_{k} \leqq \mu_{k}^{\prime}$ for all $k$, then $\mu \leqq \mu^{\prime}$.

(iii) Let $l(\mu)=n<\infty$. Then $\mu \leqq \varepsilon_{n}(s(\mu))$, and $\mu \leqq \bar{\varepsilon}_{n}(s(\mu))$ if $\mu_{0} \neq 0$.

(iv) $k \leqq n$ implies $\varepsilon_{k}(\mathfrak{m}) \leqq \varepsilon_{n}(\mathfrak{m})$ for all $\mathfrak{m}$.

(v) If $\mu \leqq \mu^{\prime}$ and $\nu \leqq \nu^{\prime}$, then $\mu+\nu \leqq \mu^{\prime}+\nu^{\prime}$.

Proof. (i) and (ii) follow from the fact that it is always possible to define operations which will not change any pre-existing subalgebra structure. Specifically, if an $n$-ary operation is required, then we do not alter subalgebra structure by taking as an operation the function $f$ defined by $f\left(x_{0}, \cdots, x_{n-1}\right)=x_{0}$. (iii) and (iv) follow from the fact 
that any operation can be replaced by one of higher rank in such a way that subalgebra structure is not affected. E.g., if $f$ is a binary operation, we can replace it by the ternary operation $g$ defined by $g\left(x_{0}, x_{1}, x_{2}\right)=f\left(x_{0}, x_{1}\right)$. To prove $(\mathrm{v})$, let $\mathfrak{A} \in T(\mu+\nu)$. Then $\mathfrak{A}=$ $S(A ; F)$, where $\langle A ; F\rangle \in K(\mu+\nu)$, and we may write $F=F_{1} \cup F_{2}$, where $\left\langle A ; F_{1}\right\rangle \in K(\mu)$ and $\left\langle A ; F_{2}\right\rangle \in K(\nu)$. Since $\mu \leqq \mu^{\prime}$ and $\nu \leqq \nu^{\prime}$, we have $S\left(A ; F_{1}\right)=S\left(A ; G_{1}\right)$ and $S\left(A ; F_{2}\right)=S\left(A ; G_{2}\right)$, where $\left\langle A ; G_{1}\right\rangle \in K\left(\mu^{\prime}\right)$ and $\left\langle A ; G_{2}\right\rangle \in K\left(\nu^{\prime}\right)$. Now,

$$
\begin{aligned}
\mathfrak{U} & =S(A ; F)=S\left(A ; F_{1}\right) \cap S\left(A ; F_{2}\right)=S\left(A ; G_{1}\right) \cap S\left(A ; G_{2}\right) \\
& =S\left(A ; G_{1} \cup G_{2}\right) \in K\left(\mu^{\prime}+\nu^{\prime}\right),
\end{aligned}
$$

whence $\mu+\nu \leqq \mu^{\prime}+\nu^{\prime}$. We note that the analogous statement and proof hold for arbitrary sums.

The following lemma provides a construction that we shall use frequently.

Lemma 1.2. Suppose $\mu$ is a multiplicity type and $n$ an integer with $0<n<\mu_{n}$. Let $A$ be a set with $|A|=\mu_{n}$, let $C \leqq A$ with $|C|=n$, and let $p \in A \backslash C$. Let $\mathfrak{A}=\{A\} \cup\{B \mid C \nsubseteq B \subseteq A\}$, and let $\mathfrak{A}_{p}=\{B \cup\{p\} \mid B \in \mathfrak{U}\}$. If $\mu_{0}=0$ then $\mathfrak{A} \in T(\mu)$, and if $\mu_{0} \neq 0$ then $\mathfrak{A}_{p} \in T(\mu)$.

Proof. Suppose $\mu_{0}=0$. For each $a \in A$ define the $n$-ary operation $f_{a}$ by:

$$
f_{a}\left(x_{0}, \cdots, x_{n-1}\right)= \begin{cases}a & \text { if }\left\{x_{0}, \cdots, x_{n-1}\right\}=C \\ x_{0} & \text { otherwise }\end{cases}
$$

for all $x_{i} \in A, i<n$. Let $F=\left\{f_{a} \mid a \in A\right\}$. Clearly

$$
\mathfrak{U}=S(A ; F) \in T\left(\varepsilon_{n}\left(\mu_{n}\right)\right) \subseteq T(\mu) \text {. }
$$

If $\mu_{0} \neq 0$, define for each $a \in A$ the $n$-ary operation $\bar{f}_{a}$ by:

$$
\bar{f}_{a}\left(x_{0}, \cdots, x_{n-1}\right)=\left\{\begin{array}{l}
f_{a}\left(x_{0}, \cdots, x_{n-1}\right) \text { if } p \neq x_{i}, i<n \\
p \text { if } p=x_{i} \text { for some } i<n,
\end{array}\right.
$$

and let $p^{*}$ be a nullary operation with value $p$. If $\bar{F}=\left\{\bar{f}_{a} \mid a \in A\right\} \cup\left\{p^{*}\right\}$, we have $\mathfrak{A}_{p}=S(A ; \bar{F}) \in T\left(\bar{\varepsilon}_{n}\left(\mu_{n}\right)\right) \subseteq T(\mu)$.

B. Characterization of $\mathfrak{A} \in T(\mu)$ for some $\mu$ of fixed length. For a restricted closure system $\mathfrak{A}$ and natural number $n$ we shall characterize what it means to have $\mathfrak{U} \in T(\mu)$ for some $\mu$ of length $n$. Since this is quite clear for $n=0$, we consider only positive $n$. 
Definition. A family $\mathfrak{B}$ of sets has the $n$-ary containment property if whenever we have $a_{0}, \cdots, a_{n-1} \in \mathbf{U}(X \mid X \in \mathfrak{B})$, then there is some $Y \in \mathfrak{B}$ with $a_{i} \in Y$ for all $0 \leqq i<n$.

Clearly a directed family has the $n$-ary containment property for all nonzero $n$. Also, we note that if $i \geqq j$, then the $i$-ary containment property implies the $j$-ary containment property.

Definition. A family $\mathfrak{A}$ is closed under n-ary union if whenever $\mathfrak{B} \subseteq \mathfrak{A}$ and $\mathfrak{B}$ has the $n$-ary containment property, then

$$
\bigcup(X \mid X \in \mathfrak{B}) \in \mathfrak{A} \text {. }
$$

Definition. Let $\mathfrak{A}$ be a restricted closure system over the nonvoid set $A . \quad 2$ is an $n$-ary closure system if whenever $\varnothing \neq B \subseteq A$ and $B=\mathrm{U}([C]|C \leqq B, 0<| C \mid \leqq n)$, then $B \in \mathfrak{A}$.

THEOREM 1.1. Let $\mathfrak{A}$ be a restricted closure system over the nonvoid set $A$, and let $n$ be a positive integer. The following are equivalent.

(i ) $\mathfrak{A} \in T\left(\varepsilon_{n}(\mathfrak{m})\right)$ for some cardinal $\mathrm{m}$.

(ii) $\mathfrak{U} \in T(\mu)$ for some $\mu$ of length $n$.

(iii) $\mathfrak{U} \in T(\mu)$ for some $\mu$ with $l(\mu) \leqq n$.

(iv) $\mathfrak{A}$ is closed under n-ary union.

(v) $\mathfrak{A}$ is an n-ary closure system.

Proof. Clearly (i), (ii), and (iii) are equivalent by Lemma 1.1. Assuming (i), we have $\mathfrak{A}=S(A ; F)$ where $\langle A ; F\rangle \in K\left(\varepsilon_{n}(\mathfrak{m})\right)$ for some m. Let $\mathfrak{B} \subseteq \mathfrak{U}$ where $\mathfrak{B}$ has the $n$-ary containment property. If $f \in F$ and $a_{i} \in \bigcup(X \mid X \in \mathfrak{B}), 0 \leqq i<n$, then $a_{0}, \cdots, a_{n-1} \in Y$ for some $Y \in \mathfrak{B}$, whence $f\left(a_{0}, \cdots, a_{n-1}\right) \in Y \subseteq \mathrm{U}(X \mid X \in \mathfrak{B})$, and so

$$
\mathrm{U}(X \mid X \in \mathfrak{B}) \in \mathfrak{A} \text {. }
$$

Thus (i) implies (iv).

Assuming (iv), let $\varnothing \neq B \cong A$ and suppose $B \supseteqq[C]$ for all $C \cong B$ with $0<|C| \leqq n$. Let

$$
\mathfrak{B}=\{[C]|C \leqq B, 0<| C \mid \leqq n\} .
$$

Then $\bigcup(X \mid X \in \mathfrak{B})=B$, and $\mathfrak{B}$ has the $n$-ary containment property, whence (iv) implies $B \in \mathfrak{A}$, and so (v) holds. Thus (iv) implies (v).

Assuming (v), we define a set $F$ of operations as follows. For each sequence $\left\langle a_{0}, \cdots, a_{n-1}\right\rangle \in A^{n}$ and each $a \in\left[\left\{a_{0}, \cdots, a_{n-1}\right\}\right]$, define an $n$-ary operation $f$ by $f\left(a_{0}, \cdots, a_{n-1}\right)=a$ and $f\left(x_{0}, \cdots, x_{n-1}\right)=x_{0}$ if $\left\langle x_{n}, \cdots, x_{n-1}\right\rangle \neq\left\langle a_{0}, \cdots, a_{n-1}\right\rangle$. 
Let $F$ be the set of all operations defined in this manner. It is clear that $\mathfrak{A} \subseteq S(A ; F)$. To verify the reverse inclusion, let $B \in S(A ; F)$ and let $C \leqq B$ with $0<|C| \leqq n$. Enumerate $C$ as a sequence $\left\langle a_{0}, \cdots, a_{n-1}\right\rangle$ (possibly with repeated terms) and let $a \in[C]$. Then, for the operation $f$ defined in terms of $\left\langle a_{0}, \cdots, a_{n-1}\right\rangle$ and $a$, we have $a=f\left(a_{0}, \cdots, a_{n-1}\right) \in B$, and so $[C] \cong B$. Applying (v) we have $B \in \mathfrak{A}$, whence $\mathfrak{U}=S(A ; F) \in T\left(\varepsilon_{n}(|F|)\right)$. Thus (v) implies (i) and the theorem is proved.

COROLlary. Let $n>0$ and let $\mu$ be a multiplicity type with $l(\mu)<n$. Then $\varepsilon_{n} \geqq \underline{\text { and }} \bar{\varepsilon}_{n} \not \leq \mu$.

Proof. The case $n=1$ is trivial, so assume $n>1$. Let $A$ be a set of cardinality $n+1, A=\left\{a_{0}, \cdots, a_{n}\right\}$, and let $A^{*}=\left\{a_{0}, \cdots, a_{n-1}\right\}$. Define an $n$-ary operation $f$ by $f\left(a_{0}, \cdots, a_{n-1}\right)=a_{n}$ and $f\left(x_{0}, \cdots, x_{n-1}\right)=$ $x_{0}$ if $\left\langle x_{0}, \cdots, x_{n-1}\right\rangle \neq\left\langle a_{0}, \cdots, a_{n-1}\right\rangle$.

Now, $A^{*}=\bigcup\left([C]\left|C \subseteq A^{*}, 0<\right| C \mid \leqq l(\mu)\right)$, but $A^{*} \notin S(A ; f)$, whence $S(A ; f)$ is not an $l(\mu)$-ary closure system, and so $S(A ; f) \notin T(\mu)$ by Theorem 1.1. Thus, $\varepsilon_{n} \not \leq \mu$. To see that $\bar{\varepsilon}_{n} \not \equiv \mu$, adjoin an element $p$ to $A$, define $f$ as above, and define also a nullary operation with the value $p$; then apply a similar argument.

C. Necessary conditions for $\mu \leqq \mu^{\prime}$. Before establishing necessary conditions for $\mu \leqq \mu^{\prime}$ we shall prove two lemmas which simplify special cases. In proving these lemmas, and elsewhere in the sequel, we shall make use of the following well-known inequality (see, e.g., [2], Chapter 1). If $\mathfrak{A}$ is a restricted closure system over the nonvoid set $A$, and $\mathfrak{U} \in T(\mu)$, then, for each nonvoid $B \subseteq A$, we have $|[B]| \leqq$ $|B| \cdot s(\mu) \cdot \boldsymbol{\aleph}_{0}$.

Lemma 1.3. ( i ) Suppose $l(\mu)>0$ and $0<\mu_{0} \leqq \boldsymbol{\aleph}_{0}$. If $\mu^{\prime}$ is defined by $\mu_{0}^{\prime}=1$ and $\mu_{i}^{\prime}=\mu_{i}$ for $i>0$, then $\mu \equiv \mu^{\prime}$.

(ii) If $\mu \leqq \mu^{\prime}$ and $s\left(\mu^{\prime}\right) \geqq \boldsymbol{\aleph}_{0}$, then $s(\mu) \leqq s\left(\mu^{\prime}\right)$.

(iii) If $\mu \leqq \mu^{\prime}$ and $s\left(\mu^{\prime}\right) \leqq \boldsymbol{\aleph}_{0}$, then $s(\mu) \leqq \boldsymbol{\aleph}_{0}$.

Proof. To prove (i) it suffices by Lemma 1.1 to show that $\mu \leqq \mu^{\prime}$. First suppose $s(\mu)>\boldsymbol{\aleph}_{0}$. Then there is some $n>0$ such that $\mu_{n}$ is infinite. For such an $n$ we have

$$
\mu=\varepsilon_{0}\left(\mu_{0}-1\right)+\mu^{\prime} \leqq \varepsilon_{n}\left(\mu_{0}-1\right)+\mu^{\prime}=\mu^{\prime} .
$$

Now suppose $s(\mu) \leqq \boldsymbol{\aleph}_{0}$ and let $\mathfrak{A} \in T(\mu) ; \mathfrak{A}=S(A ; F)$ where $\langle A ; F\rangle \in$ $K(\mu)$. Let $f$ be an $n$-ary operation for some $n>0$ (such an $f$ must exist because $l(\mu)>0$ ), let $B$ be the set of values of the nullary 
operations in $F$, and let $C=[B]$. Since $0<|B| \leqq \aleph_{0}$, we have $0<|C| \leqq \boldsymbol{\aleph}_{0}$, so we enumerate $C$ as $C=\left\{c^{0}, c^{1}, \cdots, c^{i}, \cdots\right\}$ in such a way that if $C$ is infinite then all elements in the enumeration are distinct, while if $C$ is finite, of power $N$, then $c^{i}=c^{N-1}$ whenever $i \geqq N$.

Let $F^{\prime}=\left(F \backslash\left(F_{0} \cup\{f\}\right)\right) \cup\left\{d^{0}, f^{\prime}\right\}$, where $F_{0}$ is the set of nullary operations in $F, d^{0}$ is a nullary operation with value $c^{0}$, and $f^{\prime}$ is an $n$-ary operation defined by:

$$
f^{\prime}\left(x_{0}, \cdots, x_{n-1}\right)=\left\{\begin{array}{l}
c^{i+1} \text { if } x_{0}=x_{1}=\cdots=x_{n-1}=c^{i} \in C \\
f\left(x_{0}, \cdots, x_{n-1}\right) \text { otherwise }
\end{array}\right.
$$

It is straightforward to verify that $\mathfrak{A}=S\left(A ; F^{\prime}\right) \in T\left(\mu^{\prime}\right)$, whence $\mu \leqq \mu^{\prime}$.

To prove (ii) let $A$ be a set of cardinality $s(\mu)$ and define operations $f_{a}, a \in A$, such that $S\left(A ;\left\{f_{a} \mid a \in A\right\}\right) \in T(\mu)$ and $f_{a}$ is a constant function assuming the value $a$. Then $S\left(A ;\left\{f_{a} \mid a \in A\right\}\right)=\{A\}$, so $|[\{a\}]|=s(\mu)$ for all $a \in A$. Assuming $\mu \leqq \mu^{\prime}$, we then have:

$$
|[\{a\}]| \leqq|\{a\}| \cdot s\left(\mu^{\prime}\right) \cdot \boldsymbol{\aleph}_{0}=s\left(\mu^{\prime}\right),
$$

whence $s(\mu) \leqq s\left(\mu^{\prime}\right)$.

To prove (iii) let $\mu^{*}$ be any multiplicity type such that $\mu^{\prime} \leqq \mu^{*}$ and $s\left(\mu^{*}\right)=\boldsymbol{\aleph}_{0}$. (e.g., $\left.\mu^{*}=\mu^{\prime}+\varepsilon_{1}\left(\boldsymbol{\aleph}_{0}\right)\right)$ Then $\mu \leqq \mu^{*}$, whence $s(\mu) \leqq$ $s\left(\mu^{*}\right)=\aleph_{0}$.

LeMmA 1.4. (i ) $\varepsilon_{0}(m) \leqq \varepsilon_{1}$ if $0<m<\boldsymbol{\aleph}_{0}$.

(ii) $\varepsilon_{0}(m) \leqq \bar{\varepsilon}_{1}$ if $0<m \leqq \boldsymbol{\aleph}_{0}$.

(iii) $\varepsilon_{0}\left(\boldsymbol{\gamma}_{0}\right) \geqq \varepsilon_{1}$.

(iv) $\varepsilon_{0}(n)+\varepsilon_{1}(m) \not \varepsilon_{1}(m)$, if $0<n \leqq \boldsymbol{\aleph}_{0}$ and $0<m<\boldsymbol{\aleph}_{0}$.

(v) $\bar{\varepsilon}_{1}\left(\boldsymbol{\aleph}_{0}\right) \leqq \varepsilon_{1}\left(\boldsymbol{\aleph}_{0}\right)$.

Proof. (i ) Let $\mathfrak{A} \in T\left(\varepsilon_{0}(m)\right) ; \mathfrak{A}=S(A ; F)$ where $\langle A ; F\rangle \in K\left(\varepsilon_{0}(m)\right)$. Let $n$ be the number of distinct elements of $A$ which are values of nullary operations. If $n=1$ we are through; if $n>1$, let these elements be $c_{0}, \cdots, c_{n-1}$ and define the unary operation $f$ by $f\left(c_{i}\right)=$ $c_{i+1}$ for $i<n-1$, and $f(x)=c_{0}$ for all other $x \in A$. Then $S(A ; f)=\mathfrak{A}$, whence $\mathfrak{U} \in T\left(\varepsilon_{1}\right)$, and so $\varepsilon_{0}(m) \leqq \varepsilon_{1}$.

(ii) $\varepsilon_{0}(m) \leqq \varepsilon_{0}(m)+\varepsilon_{1} \equiv \bar{\varepsilon}_{1}$ by Lemma 1.3.

(iii) Let $N$ be a set of cardinality $\aleph_{0}$ and let each element of $N$ be the value of a nullary operation. Then $N$ is the only subalgebra. Thus, if it were true that $\varepsilon_{0}\left(\boldsymbol{N}_{0}\right) \leqq \varepsilon_{1}$, there would exist a unary operation $f$ such that $S(N ; f)=\{N\}$. Under this assumption, $[\{x\}]=$ $\left\{x, f(x), f^{2}(x), \cdots, f^{n}(x), \cdots\right\}$ for each $x \in N$, and we cannot have $x=$ $f^{k}(x)$ for any $k$, since this would imply that $[\{x\}]$ is finite. Now, 
$[\{f(x)\}]=\left\{f(x), \cdots, f^{n}(x), \cdots\right\} . \quad$ But $x \notin[\{f(x)\}]$, since $x \in[\{f(x)\}]$ implies $x=f^{k}(x)$ for some $k$. Thus $[\{f(x)\}] \neq N$, a contradiction.

(iv) In view of Lemma 1.3 , it suffices to show that $\bar{\varepsilon}_{1}(m) \geqq \varepsilon_{1}(m)$. By (ii), $\varepsilon_{0}\left(\boldsymbol{S}_{0}\right) \leqq \bar{\varepsilon}_{1}$, whence it follows from (iii) that $\bar{\varepsilon}_{1} \not \varepsilon_{1}$, and so (iv) holds for $m=1$. The following proof shows that (iv) holds for $m=2$, but it will be evident that an analogous proof can be given for any finite $m \neq 0$.

Let $N$ be a set of cardinality $\boldsymbol{\aleph}_{0}$, let $x, p \in N$, and let the unary operations $f_{1}$ and $f_{2}$ be defined such that $\{x\}$ generates $N, f_{1}(x) \neq x \neq$ $f_{2}(x)$, and such that for $i, j \in\{1,2\}$ and $y, z \in N \backslash\{p\}, f_{i}(y)=f_{j}(z)$ implies $i=j$ and $y=z$. Also, we require that $f_{1}(p)=p=f_{2}(p)$ and that $p$ be the value of a nullary operation $p^{*}$. Letting $\mathfrak{U}=S\left(N ; f_{1}, f_{2}, p^{*}\right)$, it is clear that $\mathfrak{A} \in T\left(\bar{\varepsilon}_{1}(2)\right)$. Assuming $\bar{\varepsilon}_{1}(2) \leqq \varepsilon_{1}(2)$, we have unary operations $g_{1}, g_{2}$ with $\mathfrak{U}=S\left(N ; g_{1}, g_{2}\right)$. Since $p \in N=[\{x\}]$, we must have $p=h(x)$ where $h$ is a function made up a finite number of compositions of $g_{1}$ and $g_{2}$. Choosing $h$ so that the number of compositions is as small as possible, we must have $p=h(x)=g_{i}(a)$ for some $a \neq p$ and $i \in\{1,2\}$. Without loss of generality, suppose $i=1$. Since $\{p\} \in \mathfrak{U}$ we must have $g_{1}(p)=p$, and so $g_{1}^{n}(a)=p$ for all $n \geqq 1$. Therefore

$$
[\{a\}] \backslash\{p\}=\left\{a, g_{2}(a), g_{2}^{2}(a), \cdots, g_{2}^{n}(a), \cdots\right\} \quad \text { and } \quad a \neq g_{2}^{n}(a)
$$

for all $n$ because $[\{a\}]$ is infinite.

Now $[\{a\}] \backslash\{a, p\}=A_{1} \cup A_{2}$, where $A_{i}=\left[\left\{f_{i}(a)\right\}\right] \backslash\{p\}, i=1$, 2. Suppose, without loss of generality, that $g_{2}(a) \in A_{1}$. Then $A_{1} \cup\{p\} \in \mathfrak{A}$ implies $g_{2}^{n}(a) \in A_{1}$ for all $n$, but this contradicts the fact that $A_{2}$ is nonvoid.

( v ) Using Lemma 1.1, we have

$$
\bar{\varepsilon}_{1}\left(\boldsymbol{\aleph}_{0}\right)=\varepsilon_{0}+\varepsilon_{1}\left(\boldsymbol{\aleph}_{0}\right) \leqq \varepsilon_{1}+\varepsilon_{1}\left(\boldsymbol{\aleph}_{0}\right)=\varepsilon_{1}\left(\boldsymbol{\aleph}_{0}\right) .
$$

For convenience in referring to (iii) and (iv) above, let us set

$$
E_{1}=\left\{\left\langle\varepsilon_{0}(n)+\varepsilon_{1}(m), \varepsilon_{1}(m)\right\rangle \mid 0<n \leqq \boldsymbol{\aleph}_{0}, 0<m<\boldsymbol{\aleph}_{0}\right\},
$$

and let $E=E_{1} \cup\left\{\left\langle\varepsilon_{0}\left(\boldsymbol{\aleph}_{0}\right), \varepsilon_{1}\right\rangle\right\}$.

THEOREM 1.2. If $\mu \leqq \mu^{\prime}$, then the following hold:

(i) $\mu^{\prime}$ accepts $\mu$;

(ii) $l(\mu) \leqq l\left(\mu^{\prime}\right)$;

(iii) $l(\mu)=l\left(\mu^{\prime}\right)=n<\infty$ implies $\mu_{n} \leqq \mu_{n}^{\prime}$;

(iv) $\left\langle\mu, \mu^{\prime}\right\rangle \notin E$.

Proof. (i) and (iv) were proved in Lemmas 1.1 and 1.4. To prove (ii), note that by the corollary to Theorem $1.1, \varepsilon_{n} \leqq \mu^{\prime}$ implies $n \leqq$ $l\left(\mu^{\prime}\right)$ for all $n$. If $\mu_{0}=0$, then $\varepsilon_{n} \leqq \mu \leqq \mu^{\prime}$ for each $n$ such that 
$\mu_{n} \neq 0$, so it follows that $n \leqq l\left(\mu^{\prime}\right)$ for such $n$, and therefore $l(\mu) \leqq$ $l\left(\mu^{\prime}\right)$. If $\mu_{0} \neq 0$, simply replace $\varepsilon_{n}$ by $\bar{\varepsilon}_{n}$.

Since (iii) is trivial for $n=0$, we consider $n \neq 0$. First let us suppose $\mu_{0}=0$ and construct a set $A$ of cardinality $n ! \mu_{n}+n$ such that $a_{0}, \cdots, a_{n-1}$ are distinct elements of $A$, and $A \backslash\left\{a_{0}, \cdots, a_{n-1}\right\}=$ $\mathrm{U}\left(B_{T} \mid T \in S_{n}\right)$, where $S_{n}$ is the set of permutations of $\{0, \cdots, n-1\}$, and $\left|B_{T}\right|=\mu_{n}$ for each $T \in S_{n}$.

Fix some $U \in S_{n}$, and let $T^{*}: B_{U} \rightarrow B_{T}$ be a bijection, for each $T \in S_{n}$. For each $x \in B_{U}$, define an $n$-ary operation $f_{x}$ by:

$$
f_{x}\left(a_{0 T}, \cdots, a_{(n-1) T}\right)=x T^{*} \quad \text { for all } T \in S_{n},
$$

and

$$
f_{x}\left(x_{0}, \cdots, x_{n-1}\right)=x_{0} \quad \text { if } \quad\left\{x_{0}, \cdots, x_{n-1}\right\} \neq\left\{a_{0}, \cdots, a_{n-1}\right\} .
$$

Let $\mathfrak{A}=S\left(A ;\left\{f_{x} \mid x \in B_{U}\right\}\right)$. Clearly $\mathfrak{A} \in T\left(\varepsilon_{n}\left(\mu_{n}\right)\right) \subseteq T(\mu)$, and we note that $\mathfrak{X}=\{A\} \cup\left\{B \mid\left\{a_{0}, \cdots, a_{n-1}\right\} \nsubseteq B \subseteq A\right\}$. Now, $\mu \leqq \mu^{\prime}$ implies that $\mathfrak{U}=S(A ; F)$ for some $\langle A ; F\rangle \in K\left(\mu^{\prime}\right)$. For each $T \in S_{n}$, let

$$
E_{T}=\left\{x \mid x=f\left(a_{0 T}, \cdots, a_{(n-1) T}\right), f \in F, f \text { is } n \text {-ary }\right\},
$$

and let

$$
A^{*}=\left\{a_{0}, \cdots, a_{n-1}\right\} \cup \cup\left(E_{T} \mid T \in S_{n}\right) .
$$

We show that $A^{*} \in \mathfrak{Y}$. Let $x_{0}, \cdots, x_{n-1} \in A^{*}$ and $f \in F$. Then $f$ is $k$-ary for some $k$ and since $\mu^{\prime}$ accepts $\mu$ we have $k>0$. Since $l\left(\mu^{\prime}\right)=n$, we have $k \leqq n$. If $k=n$ and $\left\{x_{0}, \cdots, x_{n-1}\right\}=\left\{a_{0}, \cdots, a_{n-1}\right\}$, then $f\left(x_{0}, \cdots, x_{k-1}\right) \in A^{*}$ by construction. In all other cases, we have $\left\{x_{0}, \cdots, x_{k-1}\right\} \in \mathfrak{A}$, whence

$$
f\left(x_{0}, \cdots, x_{k-1}\right) \in\left\{x_{0}, \cdots, x_{k-1}\right\} \subseteq A^{*} .
$$

Thus $A^{*} \in \mathfrak{A}$, and since $A^{*} \supseteqq\left\{a_{0}, \cdots, a_{n-1}\right\}$, we have $A=A^{*}$, whence $n ! \mu_{n}+n=|A|=\left|A^{*}\right| \leqq n ! \mu_{n}^{\prime}+n$, and so $\mu_{n} \leqq \mu_{n}^{\prime}$.

If $\mu_{0} \neq 0$, we modify the construction as in the proof of Lemma 1.2 and the argument is similar to the above.

2. Characterization of $\mu \leqq \mu^{\prime}$. In this section we will show that the conditions of Theorem 1.2 are necessary and sufficient if the entries of $\mu$ are all countable, and that these conditions along with a fifth condition characterize $\mu \leqq \mu^{\prime}$ whenever $\mu^{\prime}$ has finite length. Moreover, we will develop "normal forms" for multiplicity types, whereby every multiplicity type will have a unique representation in normal form, and a multiplicity type of finite length in normal form will be minimal in the pointwise ordering of all multiplicity types equivalent to it. 
A. Countable multiplicity types. A multiplicity type is said to be countable if all its entries are countable. First we shall characterize $\mu \leqq \mu^{\prime}$ where $\mu$ and $\mu^{\prime}$ are countable and differ in length.

LEMma 2.1. If $\mu$ is a countable multiplicity type of finite length $n>0$, then $\mu \leqq \varepsilon_{n+1}$. Also, if $\mu_{0} \neq 0$, then $\mu \leqq \bar{\varepsilon}_{n+1}$.

Proof. Let $\mathfrak{A} \in T(\mu) ; \mathfrak{A}=S(A ; F)$ where $\langle A ; F\rangle \in K(\mu)$. Define an $(n+1)$-ary operation $f$ on $A$ as follows.

For each $x=\left\langle x_{0}, \cdots, x_{n-1}\right\rangle \in A^{n},\left[\left\{x_{0}, \cdots, x_{n-1}\right\}\right]$ is countable, so we write $\left[\left\{x_{0}, \cdots, x_{n-1}\right\}\right]=\left\{x^{0}, x^{1}, \cdots, x^{i}, \cdots\right\}$ in such a way that $x^{0}=x_{0}$ and if the set is infinite all elements in the listing are distinct, while if the set is finite, of power $N$, we require that $x^{i}=x^{N-1}$ for all $i \geqq N$.

For each $x^{i} \in\left[\left\{x_{0}, \cdots, x_{n-1}\right\}\right]$, we define $f\left(x_{0}, \cdots, x_{n-1}, x^{i}\right)=x^{i+1}$. Finally we define $f\left(x_{0}, \cdots, x_{n-1}, x_{n}\right)=x_{0}$ if $x_{n} \notin\left[\left\{x_{0}, \cdots, x_{n-1}\right\}\right]$. It is routine to verify that $\mathfrak{U}=S(A ; f) \in T\left(\varepsilon_{n+1}\right)$, whence $\mu \leqq \varepsilon_{n+1}$. The last statement now follows from Lemma 1.3.

CoROLlaRY. If $\mu$ is a multiplicity type such that $l(\mu)>0$ and $\mu \neq \varepsilon_{1}$, then $\bar{\varepsilon}_{1} \leqq \mu$.

Proof. We consider two cases.

Case 1. Suppose $l(\mu)=1$. If $\mu_{0} \neq 0$, then $\bar{\varepsilon}_{1} \leqq \mu$ by Lemma 1.1. If $\mu_{0}=0$, then $\mu \neq \varepsilon_{1}$ implies $\mu_{1}>1$, whence $\bar{\varepsilon}_{1} \leqq \varepsilon_{1}(2) \leqq \mu$.

Case 2. Suppose $l(\mu)>1$, and choose $m>1$ such that $\mu_{m} \neq 0$. If $\mu_{0} \neq 0$, we have $\bar{\varepsilon}_{1} \leqq \bar{\varepsilon}_{m} \leqq \mu$. If $\mu_{0}=0$, we have, by Lemma 2.1, $\bar{\varepsilon}_{1} \leqq \varepsilon_{2} \leqq \varepsilon_{m} \leqq \mu$.

COROLLARY. Let $\mu$ and $\mu^{\prime}$ be countable multiplicity types with $l(\mu) \neq l\left(\mu^{\prime}\right)$. Then $\mu \leqq \mu^{\prime}$ if and only if the following hold:

(i) $\mu^{\prime}$ accepts $\mu$;

(ii) $l(\mu)<l\left(\mu^{\prime}\right)$;

(iii) $<\mu, \mu^{\prime}>\neq<\varepsilon_{0}\left(\boldsymbol{\aleph}_{0}\right), \varepsilon_{1}>$.

Proof. In view of Theorem 1.2, we need show only that (i)-(iii) imply $\mu \leqq \mu^{\prime}$. Since (ii) implies that $l(\mu)$ is finite, we let $n=l(\mu)$, and consider two cases.

Case 1. Suppose $n \neq 0$. By Lemma 2.1, $\mu \leqq \varepsilon_{n+1}$, so if $m$ is any integer such that $m>n$ and $\mu_{m}^{\prime} \neq 0$, we have $\mu \leqq \varepsilon_{n+1} \leqq \varepsilon_{m} \leqq \mu^{\prime}$ if $\mu_{0}^{\prime}=0$, and $\mu \leqq \bar{\varepsilon}_{n+1} \leqq \bar{\varepsilon}_{m} \leqq \mu^{\prime}$ if $\mu_{0}^{\prime} \neq 0$.

Case 2. Suppose $n=0$. There are two possibilities.

(a) $\mu=\varepsilon_{0}\left(\aleph_{0}\right)$. By (iii), $\mu^{\prime} \neq \varepsilon_{1}$, so by the preceding corollary, $\bar{\varepsilon}_{1} \leqq \mu^{\prime}$. Now $\varepsilon_{0}\left(\boldsymbol{\aleph}_{0}\right) \leqq \bar{\varepsilon}_{1}$ by Lemma 1.4 , so $\mu \leqq \mu^{\prime}$.

(b) $\mu=\varepsilon_{0}(k)$ where $0<k<\boldsymbol{\aleph}_{0}$. Choosing $m$ as in Case 1 above, we have, using Lemma 1.4, 


$$
\varepsilon_{0}(k) \leqq \varepsilon_{1} \leqq \varepsilon_{m} \leqq \mu^{\prime} \quad \text { if } \mu_{0}^{\prime}=0
$$

and

$$
\varepsilon_{0}(k) \leqq \bar{\varepsilon}_{1} \leqq \bar{\varepsilon}_{m} \leqq \mu^{\prime} \quad \text { if } \mu_{0}^{\prime} \neq 0 .
$$

We now give necessary and sufficient conditions for $\mu \leqq \mu^{\prime}$ where $\mu$ and $\mu^{\prime}$ are both countable and have the same finite length.

Lemma 2.2. Let $\mu$ be a countable multiplicity type of finite length $n$.

(i) If $n \neq 1$, then $\mu \leqq \varepsilon_{n}\left(\mu_{n}\right)$.

(ii) If $\mu_{0}=0$, then $\mu \equiv \varepsilon_{n}\left(\mu_{n}\right)$.

(iii) If $\mu_{0} \neq 0$, then $\mu \equiv \bar{\varepsilon}_{n}\left(\mu_{n}\right)$.

Proof. All three statements are obvious for $n=0$, so assume $n \neq 0$. To prove (i), let $\mathfrak{A} \in T(\mu) ; \mathfrak{A}=S(A ; F)$ where $\langle A ; F\rangle \in K(\mu)$. Let $F_{n}$ be the set of $n$-ary operations in $F$ and let $f \in F_{n}$. Define a new $n$-ary operation $f^{\prime}$ as follows.

For each $x=\left\langle x_{0}, \cdots, x_{n-2}\right\rangle \in A^{n-1},\left[\left\{x_{0}, \cdots, x_{n-2}\right\}\right]$ is countable, so we write $\left[\left\{x_{0}, \cdots, x_{n-2}\right\}\right]=\left\{x^{0}, x^{1}, \cdots, x^{i}, \cdots\right\}$ in such a way that $x^{0}=x_{0}$ and if the set is infinite then all elements in the listing are distinct, while if the set is finite, of power $N$, then we require that $x^{i}=x^{N-1}$ for all $i \geqq N$. For each $x^{i} \in\left[\left\{x_{0}, \cdots, x_{n-2}\right\}\right]$ we define

$$
f^{\prime}\left(x_{0}, \cdots, x_{n-2}, x^{i}\right)=x^{i+1} \text {. }
$$

Finally we define

$$
f^{\prime}\left(x_{0}, \cdots, x_{n-2}, x_{n-1}\right)=f\left(x_{0}, \cdots, x_{n-2}, x_{n-1}\right)
$$

if $x_{n-1} \notin\left[\left\{x_{0}, \cdots, x_{n-2}\right\}\right]$.

Letting $F^{\prime}=\left(F_{n} \mid\{f\}\right) \cup\left\{f^{\prime}\right\}$, it is routine to verify that $\mathfrak{A}=$ $S\left(A ; F^{\prime}\right) \in T\left(\varepsilon_{n}\left(\mu_{n}\right)\right)$, whence $\mu \leqq \varepsilon_{n}\left(\mu_{n}\right)$.

If $n \neq 1$, then (ii) follows at once from (i), while (ii) is trivial if $n=1$. (iii) is a ready consequence of (ii) and Lemma 1.3.

COROLlaRY. Let $\mu$ and $\mu^{\prime}$ be countable multiplicity types with $l(\mu)=l\left(\mu^{\prime}\right)=n<\infty$. Then $\mu \leqq \mu^{\prime}$ if and only if the following hold:
(i) $\mu^{\prime}$ accepts $\mu$;
(ii) $\mu_{n} \leqq \mu_{n}^{\prime}$;
(iii) $\left\langle\mu, \mu^{\prime}\right\rangle \notin E_{1}$.

Proof. By Theorem 1.2, it suffices to show that (i)-(iii) imply $\mu \leqq \mu^{\prime}$. If $\mu$ and $\mu^{\prime}$ are compatible, the corollary is immediate from the lemma. If $\mu$ and $\mu^{\prime}$ are not compatible, then by (i) we must 
have $\mu_{0} \neq 0$, whence by the lemma, $\mu \equiv \bar{\varepsilon}_{n}\left(\mu_{n}\right)$ and $\mu^{\prime} \equiv \varepsilon_{n}\left(\mu_{n}^{\prime}\right)$. If $n \neq 1$, then the corollary follows directly from (i) of the lemma, so let us assume $n=1$. If $\mu_{1}<\mu_{1}^{\prime}$ we have $\bar{\varepsilon}_{1}\left(\mu_{1}\right) \leqq \varepsilon_{1}\left(\mu_{1}+1\right) \leqq \varepsilon_{1}\left(\mu_{1}^{\prime}\right)$, so $\mu \leqq \mu^{\prime}$. If $\mu_{1}=\mu_{1}^{\prime}=\aleph_{0}$, then $\mu \leqq \mu^{\prime}$ by Lemma 1.4. By (iii) there are no further possibilities, so the corollary is proved.

To simplify the consideration of multiplicity types having infinite length, we make the following definition.

Definition. For $n$ an integer and $m$ an infinite cardinal, the multiplicity type $\zeta_{n}(\mathfrak{m})$ is defined as follows. $\left(\zeta_{n}(\mathfrak{m})\right)_{i}=0$ for $i \leqq n$, and $\left(\zeta_{n}(\mathfrak{m})\right)_{i}=\mathfrak{m}$ for $i>n$. The multiplicity type $\bar{\zeta}_{n}(\mathfrak{m})$ is defined to be the sum of $\varepsilon_{0}$ and $\zeta_{n}(\mathfrak{m})$. $\zeta_{0}(\mathfrak{m})$ and $\bar{\zeta}_{0}(\mathfrak{m})$ will be denoted simply by $\zeta(\mathfrak{m})$ and $\bar{\zeta}(\mathfrak{m})$ respectively.

Lemмa 2.3. Let $\mu$ be a countable multiplicity type of infinite length. If $\mu_{0}=0$, then $\mu \equiv \zeta\left(\boldsymbol{S}_{0}\right)$; if $\mu_{0} \neq 0$, then $\mu \equiv \bar{\zeta}\left(\boldsymbol{S}_{0}\right)$.

Proof. Suppose $\mu_{0}=0$. Clearly $\mu \leqq \zeta\left(\boldsymbol{\aleph}_{0}\right)$. Let $I$ be the set of integers at which $\mu$ has nonzero entries, and write

$$
I=\left\{n_{0}, n_{1}, \cdots, n_{k}, \cdots\right\}
$$

such that $n_{k}<n_{k+1}$. By Lemma 2.1,

$$
\begin{aligned}
\varepsilon_{n_{k}}\left(\boldsymbol{\aleph}_{0}\right) \leqq \varepsilon_{n_{k}+1}, \text { and so } \zeta\left(\boldsymbol{\aleph}_{0}\right) & =\sum\left(\varepsilon_{k}\left(\boldsymbol{\aleph}_{0}\right) \mid k>0\right) \\
& \leqq \sum\left(\varepsilon_{n_{k}}\left(\boldsymbol{\aleph}_{0}\right) \mid k>0\right) \\
& \leqq \sum\left(\varepsilon_{n_{k+1}} \mid k>0\right) \\
& \leqq \sum\left(\varepsilon_{n_{k+1}} \mid k>0\right) \leqq \mu .
\end{aligned}
$$

If $\mu_{0} \neq 0$, we may assume by Lemma 1.3 that $\mu_{0}=1$, whence the statement follows readily from the above.

Corollary. Let $\mu$ and $\mu^{\prime}$ be countable multiplicity types of infinite length. Then $\mu \leqq \mu^{\prime}$ if and only if $\mu^{\prime}$ accepts $\mu$.

Proof. The corollary is a trivial consequence of the lemma.

Combining the previous three corollaries, we now completely describe the relations $\leqq$ and $\equiv$ among countable multiplicity types, thereby obtaining, for these multiplicity types, the converse of Theorem 1.2.

THEOREM 2.1. Let $\mu$ and $\mu^{\prime}$ be multiplicity types with $\mu$ countable. Then $\mu \leqq \mu^{\prime}$ if and only if the following hold: 
(i) $\mu^{\prime}$ accepts $\mu$;

(ii) $l(\mu) \leqq l\left(\mu^{\prime}\right)$;

(iii) $l(\mu)=l\left(\mu^{\prime}\right)=n<\infty$ implies $\mu_{n} \leqq \mu_{n}^{\prime}$;

(iv) $\left\langle\mu, \mu^{\prime}\right\rangle \notin E$.

Proof. In view of Theorem 1.2 and the three corollaries above, it suffices to show that (i)-(iv) imply $\mu \leqq \mu^{\prime}$ when $\mu^{\prime}$ is not countable. If we define $\mu^{*}$ by $\mu_{i}^{*}=\mu_{i}^{\prime}$ if $\mu_{i}^{\prime} \leqq \boldsymbol{\aleph}_{0}$ and $\mu_{i}^{*}=\boldsymbol{\aleph}_{0}$ if $\mu_{i}^{\prime}>\boldsymbol{\aleph}_{0}$, then $\mu^{*}$ is countable and (i)-(iv) hold with $\mu^{*}$ in place of $\mu^{\prime}$. Thus $\mu \leqq \mu^{*}$, whence $\mu \leqq \mu^{\prime}$ by transitivity.

CoRollary. If $\mu$ and $\mu^{\prime}$ are countable multiplicity types, then $\mu \equiv \mu^{\prime}$ if and only if the following hold:

(i) $\mu$ and $\mu^{\prime}$ are compatible;

(ii) $l(\mu)=l\left(\mu^{\prime}\right)$;

(iii) $l(\mu)=n<\infty$ implies $\mu_{n}=\mu_{n}^{\prime}$.

Thus we actually have a set of "normal forms" for countable multiplicity types. That is, every countable multiplicity type is equivalent to precisely one multiplicity type of the form $\varepsilon_{n}(m), \bar{\varepsilon}_{n}(m)$, $\zeta\left(\boldsymbol{\aleph}_{0}\right)$, or $\bar{\zeta}\left(\boldsymbol{\aleph}_{0}\right)$, and the ordering among these forms is easily observed. We shall exhibit two classes, $\mathscr{N}_{*}$ and $\mathscr{N}^{*}$, of multiplicity types, each of which will serve as a class of normal forms for all multiplicity types. Further, the class $\mathscr{N}_{*}$ will be seen to contain the countable normal forms listed above.

B. Multiplicity types of finite length and minimal normal form. We now show that the conditions of Theorem 1.2, along with a fifth condition, characterize $\mu \leqq \mu^{\prime}$ when $\mu^{\prime}$ has finite length.

THEOREM 2.2. Let $\mu$ and $\mu^{\prime}$ be multiplicity types and suppose $\mu^{\prime}$ has finite length. Then $\mu \leqq \mu^{\prime}$ if and only if the following hold:

(i) $\mu^{\prime}$ accepts $\mu$;

(ii) $l(\mu) \leqq l\left(\mu^{\prime}\right)$;

(iii) $l(\mu)=l\left(\mu^{\prime}\right)=n$ implies $\mu_{n} \leqq \mu_{n}^{\prime}$;

(iv) $\left\langle\mu, \mu^{\prime}\right\rangle \notin E$;

(v) For every $k$ such that $\mu_{k}>\boldsymbol{\aleph}_{0}$ there is a $k^{\prime} \geqq k$ with $\mu_{k} \leqq \mu_{k^{\prime}}^{\prime}$.

Proof. By Theorem 2.1, we may assume $\mu$ is not countable. To see that (i)-(v) imply $\mu \leqq \mu^{\prime}$, simply decompose each of $\mu$ and $\mu^{\prime}$ into the sum of a countable multiplicity type and an uncountable multiplicity, then apply Theorem 2.1 to the countable parts and (v) to the uncountable parts. 
Conversely, suppose $\mu \leqq \mu^{\prime}$. In view of Theorem 1.2 , we need verify only $(\mathrm{v})$. If $\mu_{0}>\boldsymbol{\aleph}_{0}$, then $\mu_{0} \leqq s(\mu) \leqq s\left(\mu^{\prime}\right)$ by Lemma 1.3 , so there must be some integer $0^{\prime}$ such that $\mu_{0} \leqq \mu_{0}^{\prime}$.

Let $k>0$ such that $\mu_{k}>\aleph_{0}$. Let $A$ be a set of cardinality $\mu_{k}$ and let $C \leqq A,|C|=k$. By Lemma 1.2, we have $\mathfrak{A} \in T(\mu)$ if $\mu_{0}=0$, where $\mathfrak{A}=\{A\} \cup\{B \mid C \varsubsetneqq B \cong A\}$. We shall assume $\mu_{0}=0$; if $\mu_{0} \neq 0$, simply choose $p \in A \backslash C$ and use in place of $\mathfrak{A}$ the family $\mathfrak{H}_{p}$ of Lemma 1.2 .

Now, $\mu \leqq \mu^{\prime}$ implies $\mathfrak{A}=S(A ; F)$ for some $\langle A ; F\rangle \in K\left(\mu^{\prime}\right)$. Let $F^{*}=\{f \mid f \in F, f$ is $m$-ary for some $m \geqq k\}$. (Note that $F^{*}$ is nonvoid by (ii).) Let $A^{*}$ denote the subalgebra generated by $C$ in the algebra $\left\langle A ; F^{*}\right\rangle$. We show that $A^{*} \in \mathfrak{A}$; clearly it suffices to show that $A^{*}$ is closed under $F \backslash F^{*}$. Let $f \in F \backslash F^{*}$; then $f$ is $m$-ary for some $m<k$, and so, for $x_{0}, \cdots, x_{m-1} \in A^{*}$, we have $\left\{x_{0}, \cdots, x_{m-1}\right\} \in \mathfrak{A}$, whence $f\left(x_{0}, \cdots, x_{m-1}\right) \in A^{*}$. Thus $A^{*} \in \mathfrak{A}$, and it follows that $A^{*}=A$. Therefore $\boldsymbol{\aleph}_{0}<\mu_{k}=\left|A^{*}\right| \leqq|C| \cdot\left|F^{*}\right| \cdot \boldsymbol{\psi}_{0}=\sum\left(\mu_{m}^{\prime} \mid m \geqq k\right)$, and so $\mu_{k} \leqq \mu_{k^{\prime}}^{\prime}$ for some $k^{\prime} \geqq k$.

We shall now begin to define normal forms which generalize those for countable multiplicity types. We shall ultimately give a complete definition of a special class $\mathscr{N}_{*}$ of multiplicity types, but for now we define only what we mean by $\mu \in \mathscr{N}_{*}$ if $\mu$ is of finite length.

Definition. If $\mu$ is of finite length, we say that $\mu \in \mathscr{N}_{*}$ if and only if $l(\mu)=0$ or the following hold:

(i) Either $\mu_{0}=s(\mu)>\mu_{i}$ for all $i>0$, or $\mu_{0} \leqq 1$; if $\mu_{0} \leqq \boldsymbol{\aleph}_{0}$, then $\mu_{0} \leqq 1$.

(ii) If $0<\mu_{i} \leqq \boldsymbol{\aleph}_{0}$, then $i=0$ or $i=l(\mu)$.

(iii) If $0<i<j$ then $\mu_{i}=0$ or $\mu_{i}>\mu_{j}$.

Note that the normal forms obtained for countable multiplicity types of finite length are members of $\mathscr{N}_{*}$.

THEOREM 2.3. Every multiplicity type of finite length is equivalent to a unique member of $\mathscr{N}_{*}$. Furthermore, if $\mu \in \mathscr{N}_{*}$ and $\mu$ has finite length, then $\mu$ is minimal in the pointwise ordering of all multiplicity types equivalent to it.

Proof. First we show the existence of the representation, and then the uniqueness. Let $\mu$ have finite length $n$. Clearly we may assume $n \neq 0$. If $\mu$ is countable, then by Lemma 2.2 we are done. So suppose $\mu$ is not countable, and let $m_{0}$ be the largest integer such that $\mu_{m_{0}}=\max \left\{\mu_{i} \mid i \geqq 0, \mu_{i}>\aleph_{0}\right\}$. Let $m_{1}$ be the largest integer such that $\mu_{m_{1}}=\max \left\{\mu_{i} \mid i>m_{0}, \mu_{i}>\boldsymbol{\aleph}_{0}\right\}$. Continue this process until it terminates, say with $m_{q}$, and let $M=\left\{m_{0}, \cdots, m_{q}\right\}$. Define $\mu_{*}$ as follows. For $i>0$, let $\left(\mu_{*}\right)_{i}=\mu_{i}$ if $i \in M$ or $i=n$, and $\left(\mu_{*}\right)_{i}=0$ 
otherwise. Finally, let

$$
\left(\mu_{*}\right)_{0}=\left\{\begin{array}{l}
0 \text { if } \mu_{0}=0 \\
1 \text { if } \mu_{0} \neq 0 \text { and } m_{1} \neq 0 \\
\mu_{0} \text { if } m_{1}=0 .
\end{array}\right.
$$

It is evident that $\mu \geqq \mu_{*} \in \mathscr{N}_{*}$. Now decompose each of $\mu$ and $\mu_{*}$ into the sum of a countable multiplicity type and an uncountable multiplicity type. Clearly the countable part will be the same in each case, so it remains to show that $\alpha \mu \leqq \sum\left(\varepsilon_{m}\left(\mu_{m}\right) \mid m \in M\right)$, where $\alpha \mu$ is defined by $(\alpha \mu)_{i}=\mu_{i}$ if $\mu_{i}>\boldsymbol{\aleph}_{0}$, and $(\alpha \mu)_{i}=0$ otherwise.

For each $k \leqq q$, we define a multiplicity type $\mu^{k}$ as follows. $\mu_{i}^{0}=\mu_{i}$ if $i \leqq m_{0}$, and $\mu_{i}^{0}=0$ otherwise. For $0<k \leqq q$, define $\mu^{k}$ by: $\mu_{i}^{k}=\mu_{i}$ if $m_{k-1}<i \leqq m_{k}$, and $\mu_{i}^{k}=0$ otherwise. Thus, for $m_{k} \in M$ it is clear that $s\left(\mu^{k}\right)=\mu_{m_{k}}$, whence $\mu^{k} \leqq \varepsilon_{m_{k}}\left(\mu_{m_{k}}\right)$ by Lemma 1.1. Therefore

$$
\begin{aligned}
\alpha \mu & =\sum\left(\varepsilon_{i}\left(\mu_{i}\right) \mid \mu_{i}>\boldsymbol{\aleph}_{0}\right) \\
& \leqq \sum\left(\mu^{k} \mid 0 \leqq k \leqq q\right) \\
& \leqq \sum\left(\varepsilon_{m}\left(\mu_{m}\right) \mid m \in M\right) \text { and so } \mu \equiv \mu_{*} .
\end{aligned}
$$

To show uniqueness, suppose $\mu$ and $\mu^{\prime}$ are members of $\mathscr{N}_{*}$ having finite length, and $\mu \equiv \mu^{\prime}$. We show that $\mu=\mu^{\prime}$. Let $n=l(\mu)$. By Theorem 1.2, $\mu$ and $\mu^{\prime}$ are compatible, $l\left(\mu^{\prime}\right)=n$, and $\mu_{n}=\mu_{n}^{\prime}$. By prior results we may assume that $n \neq 0$ and that $\mu$ is not countable. Suppose $\mu \neq \mu^{\prime}$ and let $k$ be the largest integer such $\mu_{k} \neq \mu_{k}^{\prime}$. Without loss of generality, we suppose $\mu_{k}>\mu_{k}^{\prime}$, and consider two cases.

Case 1. Suppose $k=0$ and $\mu_{0} \leqq 1$. Clearly $\mu_{0} \neq 0$, so $\mu_{0}=1$, whence $\mu_{0}^{\prime}=0$. But this contradicts the fact that $\mu$ accepts $\mu^{\prime}$.

Case 2. Suppose either $k=0$ and $\mu_{0}>1$, or $k \neq 0$. In either event, $\mu_{k}>\mu_{j}$ for all $j>k$. Noting that $\mu_{n}=\mu_{n}^{\prime}$ implies $k \neq n$, we have $\mu_{k}>\boldsymbol{\aleph}_{0}$, so Theorem 2.2 asserts the existence of an integer $k^{\prime} \geqq k$ such that $\mu_{k} \leqq \mu_{k}^{\prime}$. If $k=k^{\prime}$ this is a clear contradiction, while if $k^{\prime}>k$ then, by the maximality of $k, \mu_{k^{\prime}}^{\prime}=\mu_{k^{\prime}}<\mu_{k}$, a contradiction, whence $\mu=\mu^{\prime}$. It is evident from the construction of $\mu_{*}$ that the minimality statement holds, and so the theorem is proved.

C. Multiplicity types of infinite length. We now complete the definition of the class $\mathscr{N}_{*}$.

Definition. If $\mu$ has infinite length, we say that $\mu \in \mathscr{N}_{*}$ if and only if the following hold:

(i) Either $\mu_{0}=s(\mu)>\mu_{i}$ for each $i>0$, or $\mu_{0} \leqq 1$.

(ii) If $0<\mu_{i}<\boldsymbol{\aleph}_{0}$, then $i=0$. 
(iii) There is an integer $r=r(\mu)>0$ such that:

(iiia) For $i<r, 0<i<j$ implies $\mu_{i}=0$ or $\mu_{i}>\mu_{j}$.

(iiib) For all $i \geqq r, \mu_{i}=\mu_{r}$.

(iiic) $\mu_{r-1}>\mu_{r}$ if $r>1$.

We shall show that every multiplicity type of infinite length has a unique representation in $\mathscr{N}_{*}$.

LEMmA 2.4. Let $\mu$ and $\mu^{\prime}$ be multiplicity types and suppose that for each $i>0$ either $\mu_{i}^{\prime}=0$ or $\mu_{i}^{\prime}$ is infinite. Then $\mu \leqq \mu^{\prime}$ if and only if $\mu^{\prime}$ accepts $\mu$ and $\mu_{k} \leqq \sum\left(\mu_{i}^{\prime} \mid i \geqq k\right)$ for all $k$.

Proof. Suppose $\mu^{\prime}$ accepts $\mu$ and $\mu_{k} \leqq \sum\left(\mu_{i}^{\prime} \mid i \geqq k\right)$ for all $k$, and define $\mu^{*}$ as follows. $\mu_{k}^{*}=\sum\left(\mu_{i}^{\prime} \mid i \geqq k\right)$ for $k \neq 0$, and

$$
\mu_{0}^{*}=\left\{\begin{array}{lll}
0 & \text { if } & \mu_{0}=0 \\
s\left(\mu^{\prime}\right) & \text { otherwise . }
\end{array}\right.
$$

Clearly it suffices to show that $\mu^{*} \leqq \mu^{\prime}$. To this end, let $\mathfrak{A} \in T\left(\mu^{*}\right)$; $\mathfrak{A}=S(A ; F)$ where $\langle A ; F\rangle \in K\left(\mu^{*}\right)$. For each $k$, let

$$
F_{k}=\{f \mid f \in F, f \text { is } k \text {-ary }\} \text {. }
$$

For each $k$ such that $\mu_{k}^{*} \neq 0$, we may write $F_{k}=\bigcup\left(F_{k, i} \mid i \geqq k\right)$, where $\left|F_{k, i}\right|=\mu_{i}^{\prime}$. For each $k>0$, for each $i \geqq k$, and for each $f \in F_{k, i}$, define an $i$-ary operation $f^{i}$ by

$$
f^{i}\left(x_{0}, \cdots, x_{i-1}\right)=f\left(x_{0}, \cdots, x_{k-1}\right)
$$

for all $x_{0}, \cdots, x_{i-1} \in A$. If $\mu_{0}^{*} \neq 0$, then for each $i \geqq 0$ and for each $c \in A$ which is the value of a nullary operation in $F_{0,1}$, define an $i$ ary operation having the constant value $c$. Letting $F^{\prime}$ denote the set of operations defined in this way, it is routine to check that $\mathfrak{A}=S\left(A ; F^{\prime}\right) \in T\left(\mu^{\prime}\right)$, whence $\mu^{*} \leqq \mu^{\prime}$. The proof of the converse statement is omitted because it is very similar to the proof that $\mu \leqq \mu^{\prime}$ implies (v) in Theorem 2.2.

CoRollary. Let $\mu$ and $\mu^{\prime}$ be multiplicity types and suppose $\mu^{\prime}$ has infinite length and is a member of $\mathscr{N}_{*}$. Then $\mu \leqq \mu^{\prime}$ if and only if $\mu^{\prime}$ accepts $\mu$ and for each $k$ there is a $k^{\prime} \geqq k$ with $\mu_{k} \leqq \mu_{k^{\prime}}^{\prime}$.

Proof. Since $\mu^{\prime}$ satisfies the hypothesis of the lemma, one need only verify that for $\mu^{\prime} \in \mathscr{N}_{*}$, the second condition of the lemma is equivalent to the second condition of the corollary.

THEOREM 2.4. Every multiplicity type of infinite length is equivalent to a unique member of $\mathscr{N}_{*}$. 
Proof. First we show the existence of the representation and then the uniqueness. Since Lemma 2.3 gives the representation for countable multiplicity types of infinite length, let $\mu$ be an uncountable multiplicity type of infinite length.

If $\left\{i \mid \mu_{i}=s(\mu)\right\}$ is either void or infinite, let $\mu_{*}=\zeta(s(\mu))$ if $\mu_{0}=0$, and $\mu_{*}=\bar{\zeta}(s(\mu))$ if $\mu_{0} \neq 0$. Clearly $\mu_{*} \in \mathscr{N}_{*}$ and $\mu \equiv \mu_{*}$ by Lemma 2.4.

If, however, $0<\left|\left\{i \mid \mu_{i}=s(\mu)\right\}\right|<\boldsymbol{\aleph}_{0}$, let $m_{0}=\max \left\{i \mid \mu_{i}=s(\mu)\right\}$, and let $s_{1}(\mu)=\sum\left(\mu_{i} \mid i>m_{0}\right)$. If $\left\{i \mid \mu_{i}=s_{1}(\mu)\right\}$ is either void or infinite, define ${ }^{1} \mu$ and $\mu^{1}$ as follows.

$$
\begin{gathered}
{ }^{1} \mu_{i}=\mu_{i} \text { if } i \leqq m_{0} \text { and }{ }^{1} \mu_{i}=0 \text { if } i>m_{0} ; \\
\mu_{i}^{1}=0 \text { if } i \leqq m_{0} \text { and } \mu_{i}^{1}=\mu_{i} \text { if } i>m_{0} .
\end{gathered}
$$

Let ${ }^{1} \mu_{*}$ be the unique member of $\mathscr{N}_{*}$ equivalent to ${ }^{1} \mu$. Noting that $\mu^{1} \equiv \zeta_{m_{0}}\left(s_{1}(\mu)\right)$ by Lemma 2.4, we define $\mu_{*}={ }^{1} \mu_{*}+\zeta_{m_{0}}\left(s_{1}(\mu)\right)$, whence $\mu={ }^{1} \mu+\mu^{1} \equiv \mu_{*}$ and it is clear that $\mu_{*} \in \mathscr{N}_{*}$.

If $0<\left|\left\{i \mid \mu_{i}=s_{1}(\mu)\right\}\right|<\boldsymbol{\aleph}_{0}$, let $m_{1}=\max \left\{i \mid \mu_{i}=s_{1}(\mu)\right\}$ and let $s_{2}(\mu)=\sum\left(\mu_{i} \mid i>m_{1}\right)$. If $\left\{i \mid \mu_{i}=s_{2}(\mu)\right\}$ is void or infinite, define ${ }^{2} \mu$ and $\mu^{2}$ analogously to ${ }^{1} \mu$ and $\mu^{1}$ above, and define $\mu_{*}={ }^{2} \mu_{*}+\zeta_{m_{1}}\left(s_{2}(\mu)\right)$.

Proceed in this manner, defining $m_{k}$ and $s_{k+1}(\mu)$ if $s_{k}(\mu)$ has been defined and $0<\left|\left\{i \mid \mu_{i}=s_{k}(\mu)\right\}\right|<\boldsymbol{\aleph}_{0}$. Since a strictly decreasing sequence of cardinals must be finite, this process terminates with some $n$ such that $\mu_{*}={ }^{n} \mu_{*}+\zeta_{m_{n-1}}\left(s_{n}(\mu)\right)$.

To show uniqueness, we suppose $\mu$ and $\mu^{\prime}$ are members of $\mathscr{V}_{*}$ having infinite length, such that $\mu \equiv \mu^{\prime}$. In three steps, we show that $\mu=\mu^{\prime}$.

(A) $\mu_{i}=\mu_{i}^{\prime}$ for all $i \geqq \max \left\{r(\mu), r\left(\mu^{\prime}\right)\right\}$. For, suppose $\mu_{i} \neq \mu_{i}^{\prime}$ for such an $i$. Without loss of generality, we may assume $\mu_{i}>\mu_{i}^{\prime}$. By the corollary to Lemma 2.4, there is an $i^{\prime} \geqq i$ such that $\mu_{i} \leqq \mu_{i^{\prime}}^{\prime}$. Now $i^{\prime} \geqq i \geqq r\left(\mu^{\prime}\right)$ implies $\mu_{i^{\prime}}^{\prime}=\mu_{i}^{\prime}$, whence $\mu_{i}>\mu_{i^{\prime}}^{\prime}$, a contradiction.

(B) $r(\mu)=r\left(\mu^{\prime}\right)$. For, supposing $r(\mu) \neq r\left(\mu^{\prime}\right)$ we may assume, without loss of generality, that $r(\mu)>r\left(\mu^{\prime}\right)$. Then $r(\mu)>1$, so by (iiic) in the definition of $\mathscr{N}_{*}$, we have $\mu_{k}>\mu_{k+1}$, where $k=r(\mu)-1$. By (A), we have $\mu_{k+1}=\mu_{k+1}^{\prime}$. But, since $k \geqq r\left(\mu^{\prime}\right)$, we must have $\mu_{k+1}^{\prime}=\mu_{k}^{\prime}$. Thus $\mu_{k}>\mu_{k}^{\prime}=\mu_{j}^{\prime}$ for all $j \geqq k$, and this contradicts the corollary to Lemma 2.4.

(C) $\mu_{i}=\mu_{i}^{\prime}$ for all $i<r(\mu)=r\left(\mu^{\prime}\right)$. This can be proved by an argument almost identical to the uniqueness proof in Theorem 2.3. Combining (A), (B), and (C), we have $\mu=\mu^{\prime}$ and the theorem is proved.

3. Maximal normal form and infinitary algebras. We now define a class $\mathscr{N}^{*}$ of multiplicity types with the property that every multiplicity type has a unique representation in $\mathscr{N}^{*}$ and every member of $\mathscr{N}^{*}$ is maximal in the sense in which every member of $\mathscr{N}_{*}$ having finite length is minimal. 
DEFINITION. Given a multiplicity type $\mu$, define the multiplicity type $\mu^{*}$ by: $\left(\mu^{*}\right)_{0}=0$ if $\mu_{0}=0$, and $\left(\mu^{*}\right)_{i}=\mu_{i}+\boldsymbol{\gamma}_{0} \cdot \sum\left(\mu_{j} \mid j>i\right)$ if $i>0$ or if $i=0$ and $\mu_{0} \neq 0$. We let $\mathscr{N}^{*}$ be the class of all multiplicity types having the form $\mu^{*}$ for some $\mu$.

THEOREM 3.1. Every multiplicity type is equivalent to a unique member of $\mathscr{N}^{*}$; specifically, for every $\mu, \mu^{*}$ is greatest in the pointwise ordering of all multiplicity types equivalent to $\mu$.

Proof. Consider first a multiplicity type of the form $\varepsilon_{j}(\mathfrak{m})$. If $\mathfrak{m} \leqq \boldsymbol{\aleph}_{0}$, then $\varepsilon_{j}(\mathfrak{m}) \equiv\left(\varepsilon_{j}(\mathfrak{m})\right)^{*}$ by Theorem 2.1. If $\mathfrak{m}>\boldsymbol{\aleph}_{0}$, then $\varepsilon_{j}(\mathfrak{m}) \equiv\left(\varepsilon_{j}(\mathfrak{m})\right)^{*}$ by Lemma 1.1 .

For any $\mu$, let $\mu^{*}=\sum\left(\left(\varepsilon_{j}\left(\mu_{j}\right)\right)^{*} \mid \mu_{j} \neq 0\right)$. Then $\mu \equiv \mu^{*}$ and a straightforward computation shows that $\mu^{*}=\mu^{*}$.

To prove maximality, it suffices to show that for compatible multiplicity types $\mu$ and $\mu^{\prime}$, if $\mu^{\prime} \leqq \mu$, then $\mu_{k}^{\prime} \leqq \mu_{k}^{*}$ for all $k$. If $l\left(\mu^{\prime}\right)=l(\mu)=n<\infty$, then by Theorem $1.2 \mu_{n}^{\prime} \leqq \mu_{n}=\mu_{n}^{*}$. Thus, by Theorem 1.2, it suffices to prove that $\mu_{k}^{\prime} \leqq \mu_{k}^{*}$ for all $k<l(\mu)$.

Suppose $0<l(\mu)$ and $\mu_{0}^{\prime} \neq 0$. Then $\mu_{0} \neq 0$ because $\mu^{\prime}$ accepts $\mu$, and so $s\left(\mu^{*}\right)=\mu_{0}^{*} \geqq \aleph_{0}$, whence by Lemma $1.3, \mu^{\prime} \leqq \mu \equiv \mu^{*}$ implies $\mu_{0}^{\prime} \leqq s\left(\mu^{\prime}\right) \leqq s\left(\mu^{*}\right)=\mu_{0}^{*}$.

Now suppose $\mu_{k}^{\prime} \neq 0$, where $0<k<l(\mu)$. Then $\mu_{k}^{*} \geqq \boldsymbol{\aleph}_{0}$, so we may assume $\mu_{k}^{\prime}>\boldsymbol{Y}_{0}$. Thus $k<\mu_{k}^{\prime}$ and following the proof of (v) in Theorem 2.2, we may apply Lemma 1.2 to reach the desired conclusion.

The uniqueness statement is a direct consequence of the maximality statement and the easily observed fact that $\mu=\mu^{*}$ for all $\mu \in \mathscr{N}^{*}$. Thus the theorem is proved.

Finally we mention that many of our preliminary results can easily be extended to the case of multiplicity types of algebras having infinitary operations. Using methods similar to those presented here, the author has found maximal normal forms for a wide class of infinitary multiplicity types. However, the problem of characterizing the ordering and finding normal forms for all infinitary multiplicity types remains open.

\section{REFERENCES}

1. G. Birkhoff and O. Frink, Representations of lattices by sets, Trans. Amer. Math. Soc. 64 (1948), 299-316.

2. G. Grätzer, Universal algebra, D. Van Nostrand Company, Princeton, N. J., 1968.

Received June 12, 1967. These results are taken from the author's doctoral thesis, supervised by Prof. G. Grätzer at The Pennsylvania State University. 



\section{PACIFIC JOURNAL OF MATHEMATICS}

\section{EDITORS}

\section{H. ROYDEN}

Stanford University

Stanford, California

R. R. Phelps

University of Washington

Seattle, Washington 98105

\section{J. DugundJI}

Department of Mathematics

University of Southern California

Los Angeles, California 90007

RICHARD ARENS

University of California

Los Angeles, California 90024

\section{ASSOCIATE EDITORS}
E. F. BeCKENBACH
B. H. NeumanN
F. WOLF
K. YosIDA

\section{SUPPORTING INSTITUTIONS}

\author{
UNIVERSITY OF BRITISH COLUMBIA \\ CALIFORNIA INSTITUTE OF TECHNOLOGY \\ UNIVERSITY OF CALIFORNIA \\ MONTANA STATE UNIVERSITY \\ UNIVERSITY OF NEVADA \\ NEW MEXICO STATE UNIVERSITY \\ OREGON STATE UNIVERSITY \\ UNIVERSITY OF OREGON \\ OSAKA UNIVERSITY \\ UNIVERSITY OF SOUTHERN CALIFORNIA
}

\author{
STANFORD UNIVERSITY \\ UNIVERSITY OF TOKYO \\ UNIVERSITY OF UTAH \\ WASHINGTON STATE UNIVERSITY \\ UNIVERSITY OF WASHINGTON \\ AMERICAN MATHEMATICAL SOCIETY \\ CHEVRON RESEARCH CORPORATION \\ TRW SYSTEMS \\ NAVAL WEAPONS CENTER
}

Mathematical papers intended for publication in the Pacific Journal of Mathematics should be in typed form or offset-reproduced, double spaced with large margins. Underline Greek letters in red, German in green, and script in blue. The first paragraph or two must be capable of being used separately as a synopsis of the entire paper. It should not contain references to the bibliography. Manuscripts, in duplicate if possible, may be sent to any one of the four editors. All other communications to the editors should be addressed to the managing editor, Richard Arens, University of California, Los Angeles, California 90024.

Each author of each article receives 50 reprints free of charge; additional copies may be obtained at cost in multiples of 50 .

The Pacific Journal of Mathematics is published monthly. Effective with Volume 16 the price per volume (3 numbers) is $\$ 8.00$; single issues, $\$ 3.00$. Special price for current issues to individual faculty members of supporting institutions and to individual members of the American Mathematical Society: $\$ 4.00$ per volume; single issues $\$ 1.50$. Back numbers are available.

Subscriptions, orders for back numbers, and changes of address should be sent to Pacific Journal of Mathematics, 103 Highland Boulevard, Berkeley 8, California.

Printed at Kokusai Bunken Insatsusha (International Academic Printing Co., Ltd.), 7-17, Fujimi 2-chome, Chiyoda-ku, Tokyo, Japan.

PUBLISHED BY PACIFIC JOURNAL OF MATHEMATICS, A NON-PROFIT CORPORATION

The Supporting Institutions listed above contribute to the cost of publication of this Journal, but they are not owners of publishers and have no responsibility for its content or policies. 


\section{Pacific Journal of Mathematics}

\section{Vol. 26, No. $3 \quad$ BadMonth, 1968}

Leonard Asimow, Universally well-capped cones ................. 421

Lawrence Peter Belluce, William A. Kirk and Eugene Francis Steiner,

Normal structure in Banach spaces ..................... 433

William Jay Davis, Bases in Hilbert space.................... 441

Larry Lee Dornhoff, p-automorphic p-groups and homogeneous

algebras..................................... 447

William Grady Dotson, Jr. and W. R. Mann, A generalized corollary of the

Browder-Kirk fixed point theorem ....................... 455

John Brady Garnett, On a theorem of Mergelyan ................. 461

Matthew Gould, Multiplicity type and subalgebra structure in universal

algebras............................................. 469

Marvin D. Green, A locally convex topology on a preordered space . . . . . 487

Pierre A. Grillet and Mario Petrich, Ideal extensions of semigroups . . . . . . 493

Kyong Taik Hahn, A remark on integral functions of several complex

variables ... . . . . . . . . . . . . . . . . . . . . . . . . . . . . . . . . . . . 509

Choo Whan Kim, Uniform approximation of doubly stochastic

operators..................................... 515

Charles Alan McCarthy and L. Tzafriri, Projections in $\mathscr{L}_{1}$ and

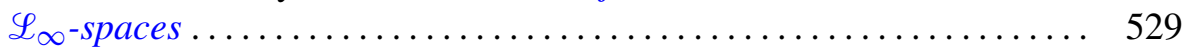

Alfred Berry Manaster, Full co-ordinals of RETs ................ 547

Donald Steven Passman, $p$-solvable doubly transitive permutation

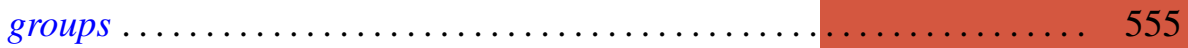

Neal Jules Rothman, An $L^{1}$ algebra for linearly quasi-ordered compact

semigroups ....................................... 579

James DeWitt Stein, Homomorphisms of semi-simple algebras .......... 589

Jacques Tits and Lucien Waelbroeck, The integration of a Lie algebra

representation ...............................

David Vere-Jones, Ergodic properties of nonnegative matrices. II ........ 601

Donald Rayl Wilken, The support of representing measures for $R(X) \ldots \ldots 621$

Abraham Zaks, Simple modules and hereditary rings .... . . 